\title{
Justice et injustice du tanfîl : la part du légal et du conventionnel dans la construction sociale du sens ordinaire du juste *
}

\section{Mohamed Nachi **}

\section{Résumé}

Dans cet article, nous proposons, d'un point de vue socio-anthropologique, une approche comparative du sens ordinaire de la justice à travers l'analyse d'un cas paradigmatique de partage selon le mode du tanfîl. Nous commençons par examiner les deux significations principales, religieuse et coutumière, de cette institution ainsi que les différentes modalités de sa mise en œuvre. Puis, à partir d'une analyse détaillée des formes de justification et des jugements déployés par des Tunisiens et des Français à propos de ce cas de partage, nous montrons comment les positions, aussi bien des uns que des autres, oscillent entre deux conceptions de la justice : d'après la première, il y a une adéquation entre le légal et le juste tandis que, selon la seconde, il y a au contraire inadéquation entre les deux. Dans le premier cas, on fait systématiquement recours à la loi comme référentiel de justification au détriment des normes conventionnelles, dans le second, c'est l'inverse qui se produit. Enfin, nous verrons, à travers l'exigence de compromis, qu'en définitive le sens de la justice se situe entre les deux.

Légalité - Normes conventionnelles - Sens ordinaire de la justice - Tanfîl.

\section{Summary}

Justice and Injustice with Regard to Tanfîl : Legality and Conventional Norms in the Making of the Ordinary Sense of J ustice

In this article, we propose a comparative approach of the ordinary sense of justice, from a socio-anthropological point of view, through the analysis of a paradigmatic case of sharing according to the mode of tanfîl. We begin with the examination of the two principal meanings of this institution, religious and customary, as well as its different modes of implementation. Then, from this detailed analysis of the forms of justifications and judgements displayed by the Tunisians and the French concerning this case of sharing, we show how either of these positions, wavers between two concepts of justice : under the first one, there is an accord between
L'auteur

Juriste, politiste et ethnologue. Chargé de cours en ethnologie à I'Université Montpellier III et membre du Groupe de sociologie politique et morale (EHESS/CNRS). II poursuit une recherche comparative sur les sentiments de justice et d'injustice en Tunisie et en France.

* Une partie de la recherche sur laquelle est basé cet article a fait l'objet d'une communication à la faculté de droit de Rabat et à I'Institut de recherches sur le Maghreb contemporain (IRMC, Maroc) à l'occasion d'un séminaire animé par Alain Roussillon sur les « Sociétés en réforme ».

** Las Rébès,

Bâtiment $2 \mathrm{D}$,

556 avenue Louis Ravas, F-34080 Montpellier. 


\section{Nachi}

Justice et injustice du tanfîl : la part du légal et du conventionnel dans la construction sociale du sens ordinaire du juste

1. Pour une définition de cette notion capitale, voir M. NACHI, Anthropologie du jugement, Paris, éd. du Cerf (à paraître). 2. Dans le cadre de cet article, eu égard aux limites qui lui sont imparties, nous ne pouvons présenter les aspects méthodologiques relatifs à notre recherche sur le terrain. Disons, très rapidement, que pour le bon déroulement de nos différentes enquêtes successives en Tunisie et en France, nous avons pu associer une démarche de type qualitatif (enquête sur le terrain, observation directe des disputes ou conflits, recueil des récits) à une approche de type quantitatif puisque nous avons dû élaborer un protocole de questionnaire (20 questions pour chaque récit) afin de pouvoir faire une analyse statistique des jugements et procéder à des croisements entre ceux des Tunisiens et ceux des Français. Ajoutons que les récits sont recueillis auprès de Tunisiens vivant en Tunisie et/ ou en France et relatent une histoire d'injustice au sens ordinaire, banal, du terme. Pour notre cas d'espèce, il faut savoir qu'il a été soumis au jugement de 127 personnes. Pour une présentation détaillée des questions de méthode, nous renvoyons au livre de M.-C. Foblets dont la démarche est à plusieurs égards assez proche de la nôtre : Les familles maghrébines et la justice en Belgique : anthropologie juridique et immigration, Paris, Karthala, 1994, p. 73-143. 3. L. BOLTANSKI et L. THÉVENOT, De la justification : les économies de la grandeur, Paris, Gallimard, 1991.

4. Cf. M. NACHI, « Le juste entre I'universel et le contextuel », Social Science Information/ Information sur les sciences sociales, 35 (2), 1996, spécialement p. 413-416. the legal and the just, whereas under the second, there is, on the contrary, a disparity between the two. In the first case, there is a systematic recourse to the law as a referent of justification to the detriment of the conventional norms, in the second case, the opposite occurs. Finally, we show how the demands of compromise in fact dictate that the sense of justice lies between the two.

Conventional norms - Legality - Ordinary sense of justice - Tanfîl.

Nous nous proposons d'étudier le sens ordinaire du juste à travers l'analyse d'un cas concret de partage d'héritage sur le mode du tanfîl, et plus précisément à travers l'examen des modalités de jugement et de justification mobilisées pour apprécier, évaluer ce cas. Partant d'un cas paradigmatique de partage, dans lequel le tanfîl est mis en œuvre et contesté, nous cherchons à déterminer la part du légal et du conventionnel dans la construction des jugements. Si l'exemple en question est propre à la Tunisie, les principes de justice mis en jeu dépassent le cadre étroit de cette société. Cette affaire permet de s'en rendre compte. Elle a été soumise à I'appréciation et au jugement ${ }^{1}$ de Tunisiens, mais aussi de Français, en vue de repérer les cas où les jugements qu'ils portent sur son dénouement convergent et les cas où ils divergent ${ }^{2}$.

Les jugements émis à propos du partage d'héritage réalisé par recours au tanfîl sont très révélateurs de la manière dont des Tunisiens et des Français se positionnent par rapport à des pratiques coutumières, à des normes légales et à des principes moraux en invoquant des référentiels normatifs qui, à leurs yeux, justifient l'idée qu'ils se font du juste. Les ordres de jugement et de justification font apparaître deux références récurrentes fondamentales : la première renvoie à la nature domestique des formes de justification, la seconde à leur nature civique ${ }^{3}$. Ces deux référentiels majeurs, mobilisés tant par les Tunisiens que par les Français, ne sont pas sans rappeler les deux conceptions philosophiques de la justice, procédurale et substantielle ${ }^{4}$. Ces deux conceptions se trouvent exprimées, sous des formulations légèrement différentes, aussi bien par les uns que par les autres. Ainsi, certains font prévaloir la primauté du légal, du formel, dans la définition du juste tandis que d'autres accordent la priorité à la dimension conventionnelle, substantielle pourrait-on dire, de la justice. À propos de la première conception, nous ne pouvons nous empêcher de souligner l'adéquation entre le légal et le juste ${ }^{5}$. S'agissant de la seconde, en revanche, force est de constater l'inadéquation entre le légal et le juste, ainsi que le recours à des appuis conventionnels

5. Pour une réflexion approfondie sur le rapport entre le juste et le légal, voir W. SADURSKI, Giving Desert Its Due : Social J ustice and Legal Theory, Dordrecht (Holland), D. Reidel Publishing Company, 1985, 329 p. 
pour légitimer le juste. La notion de «convention » doit dès lors être située en rapport direct avec ce que J ean-Pierre Dupuy nomme la «common knowledge ${ }^{6}$ ». La question est alors de savoir comment s'articulent ces deux conceptions et quelle place occupe chacune d'elles dans les répertoires de jugement et dans les modalités de justification. En d'autres termes, quelle est la part du légal et la part du conventionnel dans la construction sociale du sens ordinaire de la justice.

Notre perspective ne se préoccupe pas du droit positif : ce qui retient davantage notre attention, c'est le point de vue des gens eux-mêmes sur le juste et l'injuste ${ }^{7}$ ainsi que la manière dont ils interprètent, utilisent ou manipulent les normes en général, juridiques (le droit positif), sociales ou morales. C'est en ce sens que nous préférons parler, à la suite de $P$. Pharo, de droit ordinaire ${ }^{8}$. Dès lors, il s'agira dans notre cas d'espèce de voir dans quel contexte on invoquera les règles de droit et dans quel autre on fera prévaloir une pratique conventionnelle ou des principes moraux. Par ailleurs, à partir du moment où l'on demande à des personnes de procéder à l'appréciation du caractère juste ou injuste du dénouement de l'affaire, nous ne pouvons pas ne pas placer la catégorie du jugement au cœur de notre dispositif théorique ${ }^{9}$.

Nous allons d'abord tenter de caractériser le tanfîl avant de nous livrer successivement à l'analyse des deux grandes conceptions du juste, la légale puis la conventionnelle.

\section{Une pratique surérogatoire coutumière à caractère conventionnel}

Le mot nafl (nom) ou tanfîl (substantif), qu'on peut rendre par «acte surérogatoire », est issu de la racine $n-f-l$. Celle-ci possède essentiellement deux acceptions : la première, la nâfîla, peut être qualifiée de religieuse et tire ses fondements du Coran et de certains hadîth, alors que la seconde, le tanfîl, a trait à des pratiques coutumières. Mais dans les deux cas, on a affaire à la même racine et au même contenu sémantique: «donner quelque chose gratuitement », «en plus ». De cette racine est née le mot nâfîla, dont l'examen est particulièrement intéressant en raison des parentés repérables dans les significations de ces deux mots tanfîl et nâfîla.

Le mot nâfîla (pluriel nawâfîl) est mentionné deux fois dans le Coran pour montrer l'importance des œuvres surérogatoires, sous forme de prières notamment (salât al-nawâfîl), pour tout musulman qui veut s'assurer du pardon (samâh) de Dieu. Plusieurs hadîth (traditions prophétiques) sont venus confirmer l'importance de la nâfîla et souligner le rôle que peut jouer une telle œuvre pour qu'un musulman puisse se racheter. Dans les hadîth, le terme nafl est fréquemment utilisé surtout pour faire valoir l'intérêt auprès
Droit et Société 39-1998

6. J.-P. DupuY, « Convention et common knowledge », Revue économique, 40 (2), 1989, p. 361-400. La «théorie des conventions » se trouve depuis quelques années au cœur d'un débat mené tant par les philosophes que par les économistes et les sociologues. Pour une mise au point utile et une évaluation de la portée heuristique de la réflexion sur la question des conventions, il convient de se référer aux deux numéros spé ciaux de revues consacrés au sujet: Revue économique, 40 (2), 1989 sur I' «Économie des conventions » et Réseaux 62, 1993, dossier et forum : « Les conventions ». Nous nous sommes par ailleurs inspirés des références suivantes : Stanley CAVELL, « Le naturel et le conventionnel », dans S. CAVELL, Les voix de la raison, Paris, Seuil, coll. «L'ordre philosophique », 1996, p. 143-

199 ; Peter WINCH, « Nature and Convention », dans P. WINCH, Ethics and Action, London, Routledge \& Kegan Paul, 1972, p. 50-

72 ; Stuart HAMPSHIRE, « Morality and Convention », dans A. SEN and B. WILLIAMS (eds), Utilitarianism and Beyond, Cambridge, Cambridge University Press, 1982, p. 145-157.

7. D. MILLER, «Distributive Justice: What the People Think », Ethics, 102 (3), p. 555-593. 8. P. PHARO, « Le droit ordinaire comme morale ou commerce civil », dans F. CHAZEL et J. Commaille (dir.), Normes juridiques et régulation sociale, Paris, LGDJ, coll. «Droit et Société », 1991, p. 243-255. En outre, sur le rôle que peut jouer la phiIosophie analytique dans I'élaboration d'une approche ou d'une théorie qui met au premier plan l'usage juridique du langage ordinaire, il convient de consulter l'étude que consacre H.P. VISSER'T HoofT à ce sujet : « La philosophie du langage ordinaire et le droit », Archives de philosophie du droit, 17, 1972 p. 261-284.

9. L. THÉVENOT, « Jugements ordinaires et jugements de droit », Annales ESC, ${ }^{\circ} 6,1992$, p. 1279-1299. 
M. Nachi

Justice et injustice du tanfîl : la part du légal et du conventionnel dans la construction sociale du sens ordinaire du juste de Dieu des prières surérogatoires (salât al-nâfîla). Outre cette vertu essentielle permettant à celui qui réalise des œuvres surérogatoires de bénéficier de l'amour de Dieu et de sa compassion (rahma), les théologiens musulmans ont attribué à de telles œuvres un rôle non moins important : l'expiation de péchés légers.

Pour sa part, le tanfîl est un acte surérogatoire visant à se rapprocher le plus possible d'un partage juste. Si, dans le cas de la nâfîla, on vise à conquérir l'amour de Dieu et à s'assurer de son pardon, dans celui du tanfîl, en revanche, on tend à atteindre une certaine idée du juste en donnant quelque chose de plus pour que les choses soient, en quelque sorte, plus justes. Le tanfîl s'avère alors être au service de certains principes moraux et, d'une certaine façon, au service d'une éthique fondamentale. En ce sens, la distinction entre les deux se révèle quelque peu factice.

Il faut noter que le tanfîl était une pratique très fréquente, qui reste assez répandue, même si, de nos jours, elle peut revêtir d'autres formes. Si autrefois, à l'occasion d'une distribution, on pouvait plus facilement renoncer à une partie de sa part au profit de l'un des partenaires pour davantage « équilibrer » le partage, on a tendance actuellement à recourir plutôt à des moyens de compensation en argent. Cependant, le principe de base et le procédé restent les mêmes, en ce sens que, dans tous les cas, on cherche à opérer un partage juste en attribuant «un plus », ce quelque chose qu'on donne «gratuitement » ou presque, à celui qui a la part la moins «bonne».

C'est à ce titre que le tanfîl, en tant qu'acte de justice corrective, trouve pleinement sa place dans une réflexion générale sur le sens du juste. À ce même titre, il peut être très révélateur de la manière dont les gens pratiquent et pensent certains principes de justice.

D'où le tanfîl tire-t-il son «potentiel normatif » et sa légitimité sociale? Sans pouvoir aborder le rapport entre le droit musulman (fiqh) et la coutume ('urf), disons très rapidement que les deux ne sont pas inconciliables et qu'il ne faut surtout pas les opposer. II convient également d'ajouter que la dimension normative des pratiques sociales a toujours été intégrée, moyennant certaines exigences, dans le corpus légal constitutif du fiqh. Ainsi, pour B. Johansen, qui traite du droit hanéfite: «Parmi les sources non juridiques et non religieuses des matériaux et des règles du fiqh, la pratique sociale a toujours été acceptée et reconnue par les juristes musulmans à condition que les normes qui en résultent soient sélectionnées par les juristes, subordonnées à leur examen critique, soumises à leurs techniques de raisonnement et à leurs règles de preuve. C'est-à-dire que les dimensions normatives de la pratique sociale n'ont pas automatiquement de valeur juridique. Elles acquièrent cette valeur seulement si elles s'avèrent compatibles 
avec, d'un côté, le système du figh et, de l'autre, la justice des qadis ${ }^{10}$. »

Par ailleurs, il ne faut pas non plus opposer la coutume au droit positif, dans la mesure où celui-ci conserve les empreintes de celle-là. Tous les deux ne sont, en définitive, que la cristallisation d'un ensemble de règles et de principes ainsi que l'expression de certaines valeurs. Nous verrons plus loin que le recours à l'un ou à l'autre, en tant que système normatif de justification, prend un caractère particulier selon le mode d'argumentation déployé et selon le contexte, mais que les deux sont omniprésents et constituent incontestablement des répertoires dans lesquels les acteurs puisent leurs justifications au gré des situations ${ }^{11}$. II convient dès lors de nuancer ces multiples oppositions et de relativiser la portée de ces distinctions. Comme le fait remarquer G. Lenclud, «ce faisant, nous réintroduirons dans la discussion la perspective historique, celle du mouvement et des chevauchements, sans point de rupture absolue, sans évolution linéaire ${ }^{12} \gg$.

C'est cette perspective que nous nous proposons d'adopter en plaçant le tanfîl dans le contexte de son usage ${ }^{13}$ et en abordant son potentiel normatif du point de vue des acteurs eux-mêmes, à travers les formes de justification qu'ils mettent en œuvre. II s'agit pour ainsi dire de voir ce que le «bon sens » nous enseigne, de prime abord, sur cette institution. Et, pour commencer, il est indispensable de citer la partie du récit où le narrateur explique ce qu'il faut entendre par tanfîl. En voici un résumé succinct :

Dans les années soixante, le père de Tarek, Deli, Kamel et Rachid, ouvrier agricole, achète un lopin de terre qu'il ne peut pas exploiter lui-même. Il décide alors de le partager entre ses quatre fils. Ces derniers font venir un amîn qui procède au partage selon la pratique du tanfîl en tenant compte de la nature et de l'emplacement des différentes parts. Kamel se porte volontaire pour acquérir la partie la moins «bonne» mais aussi la plus grande. Chacun exploite sa part sans titre de propriété jusqu'au jour où un conflit éclate entre Tarek et Deli.

L'affaire est portée devant le tribunal qui, pour compenser le fait que Kamel avait eu une portion « en plus » lors du premier partage, oblige celui-ci à verser une somme d'argent à ses frères, surtout à Deli. Ce dernier accepte cet argent et Kamel se trouve dans l'obligation, faute de moyens financiers pour payer cette dette, de vendre un autre lopin de terre hérité de sa mère. Vexé, il décide alors de le vendre à un acquéreur «étranger » à la famille. Mais Rachid s'y oppose fermement au point d'intenter une action en justice pour faire annuler la vente. II obtient gain de cause, fait annuler le contrat et oblige son frère Kamel à lui vendre la parcelle en question.

Voici maintenant quelques séquences du récit. Le narrateur (Tarek) :

10. B. JOHANSEN, « Coutumes locales et coutumes universelles : aux sources des règles juridiques en droit musulman hanéfite », Annales islamologiques, XXVII, 1993, p. 29.

11. Au sujet de la notion de « répertoire normatif », voir B. DUPRET, «Représentations des répertoires juridiques en Égypte : limites d'un consensus », Monde arabe/ Maghreb-Machrek, 151, 1996, p. 32-40.

12. G. LENCLUD, « L'opposition coutume-droit dans les systèmes de reproduction », Droit et Cultures, 6, 1983, p. 8.

13. La notion de « contexte » en anthropologie juridique a été bien analysée par J.-L. AMSELLE, « Le droit contextualisé », Cahiers d'études africaines, 124, XXXI (4), 1991, p. 553-556. 
M. Nachi

Justice et injustice du tanfîl : la part du légal et du conventionnel dans la construction sociale du sens ordinaire du juste

14. Au sujet de la notion de haqq (droit), en tant que catégorie éthico-juridique fondamentale, il convient de se reporter à l'analyse décisive que lui consacre Cl. GeERTZ, Savoir local, savoir global : les lieux du savoir, Paris, PUF, coll.

«Sociologie d'aujourd'hui », 1986, spécialement p. 232-236.

15. La dénomination de la personne compétente n'est pas fortuite : ce n'est pas par hasard qu'elle est appelée amîn. Ce terme veut dire « confiant » au double sens de sûr (être sûr de soi, agir avec assurance et en toute quiétude) et de faire totalement confiance à quelqu'un. Amîn signifie donc «sûr », «en qui on peut se fier ». C'est aussi celui à qui I'on a confié quelque chose : amâna (« bien confié »), d'où, par exemple, amîn mâl (trésorier).
«[...]Le partage de cette terre, à l'époque encore en friche, s'est très bien passé. Nous avons fait appel à un amîn [souvent shaykh ou 'adûl (huissier) quelque fois bannây (maçon)], c'est dans tous les cas un expert en la matière, qui, comme le voulait la coutume ('âda), ne s'est pas limité à une distribution arithmétique de la superficie du champ mais a utilisé la pratique du tanfîl. Il a pris en compte la nature et la qualité du sol dans les différents endroits du terrain. D'autres considérations interviennent aussi dans I'usage du tanfîl : on tient compte de l'emplacement des parts - près ou loin du puits, situées à proximité d'une route ou non, etc. - et de la possibilité ou non de les irriguer.

L'amîn a remarqué, en parcourant le champ, qu'il y avait des disparités entre plusieurs endroits. II a voulu alors attribuer, équitablement, "à chacun son dû" [habb yensof wa ye'dîl bâsh ya'atî kul wâhid haqqu].

La technique du tanfîl est la mieux adaptée à ce genre de situation. C'est un procédé qui permet de donner plus de superficie à celui qui va avoir la parcelle la moins avantageuse. Mes frères ne voyaient aucune objection, ils étaient satisfaits.

L'amîn a délimité la partie la moins intéressante et nous a expliqué comment cette part, défavorable sur le plan de la qualité, serait privilégiée quantitativement. II a alors demandé s'il y avait parmi nous un volontaire pour recevoir cette part, sinon, il procéderait par tirage au sort. Mon frère Kamel, d'emblée d'accord, s'est porté volontaire et a accepté la parcelle de terre la moins fertile et la mieux exposée puisqu'elle donne sur une route principale.

Moi et mes deux autres frères, nous avons approuvé le partage. Nous étions donc d'accord pour que l'amîn attribue à Kamel une surface en plus en contrepartie de son choix défavorable. Ce surplus de

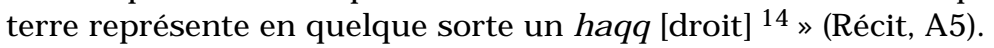

On doit relever le rôle et la responsabilité considérables dont jouit la personne chargée d'effectuer le partage, l'amîn en l'occurrence ${ }^{15}$. II faut en effet toujours l'intervention d'une personne compétente qui, disposant d'un savoir spécifique et, pour ainsi dire, d'une certaine grandeur, peut arriver à faire adopter la solution la plus juste en usant d'une certaine sagesse pratique et d'un certain sens de l'ijtihâd. Cette personne peut tirer sa compétence d'un savoir religieux (imâm) ou juridique ('adûl ou huissier) et souvent des deux à la fois. Elle peut en disposer aussi par la maîtrise d'une technique ou d'une pratique spécifiques (artisan, maçon, paysan). Il faut savoir aussi que parmi ces personnes compétentes, nombreuses sont celles qui, conscientes de la charge qui leur incombe, refusent de remplir cette fonction par peur d'être à l'origine d'un zulm (injustice), car, arguent-elles, «seul Dieu peut être toujours 'âdil (juste) parce qu'il est parfait ». Dans tous les cas, cette compétence doit être conventionnellement reconnue et socialement attestée.

À côté de ces emplois larges du mot, on trouve une acception plus technique selon laquelle l'amîn se trouve investi d'une fonc- 
tion « de confiance», d'une charge qui engage sa responsabilité et son sens de l'honneur. Le signe correspond donc parfaitement à la « chose ». L'amîn est celui qui est chargé de procéder à un partage selon des normes implicites conventionnelles communément partagées mais dont il a l'entière maîtrise. Ce sont ses qualités intrinsèques et leur reconnaissance par sa communauté qui font la grandeur de l'amîn et le rendent plus à même que quiconque d'effectuer l'appréciation qui convient et d'opérer ensuite un partage juste. Pour notre cas d'espèce, le récit le montre bien, l'amîn a dû avant toutes choses se rendre compte des disparités entre différents parcelles du terrain pour pouvoir ensuite déterminer la superficie adéquate qui sera attribuée «en plus » (munafîla). Cela afin, d'une part, de donner «à chacun son dû » (haqqu) 16 et, d'autre part, d'éviter d'éventuels sentiments d'injustice.

C'est parce qu'il n'a pas une procédure préétablie à suivre, dont la simple application permettrait la réalisation d'un partage juste, que l'amîn se trouve être amené à faire un effort particulier d'interprétation de la situation, un ijtîhâd, pour trouver la solution la mieux adaptée, la plus convenable. Il faut à cet égard souligner le caractère créatif, inventif, de sa démarche. Il s'agit d'une investigation de type casuistique, quasi prétorienne visant au partage le plus juste dans le cas d'espèce. Non seulement l'amîn doit mettre en application sa compétence, mais il doit aussi mobiliser un système de valeurs, de principes moraux et, en un sens, une certaine idée du juste pour que personne ne soit lésé par sa décision. De manière constructive, là où c'est possible, il doit faire passer en priorité l'idée de justice, même si dans certains cas les enjeux sociaux sont tels et la situation tellement inextricable qu'il a beaucoup de mal à se faire entendre.

On dit du tanfîl qu'il se pratique « 'alâ al-ma'rûf » (selon ce qui est [re]connu et partagé conventionnellement) ou «'alâ al-ma'mûli bihi » ou «'âda » (selon ce qui est communément pratiqué, ou coutume). Le terme «al-ma'rûf » nous renvoie à celui de « 'urf » qui veut dire «pratique reconnue et utilisée », c'est-à-dire «'âda ». Les deux expressions sont synonymes ${ }^{17}$. On observera la dimension pragmatique du tanfîl, car c'est dans sa mise en œuvre, dans la pratique, que cette institution trouve sa meilleure expression. Comme l'écrit justement P. Ricœur : «Une institution considérée comme règle de distribution n'existe que pour autant que les individus y prennent part ${ }^{18}$. »

Elles nous montrent enfin l'importance de la dimension conventionnelle sans laquelle le tanfîl n'aurait aucune effectivité pratique.

II n'est pas exagéré de parler d'une pratique coutumière ('urf) qui, à défaut d'établir un système normatif canonique, indique l'orientation à suivre, montre l'attitude à adopter pour réparer une injustice ou corriger un droit. Ainsi, si la nâfîla permet, comme

16. Cl. GeERTZ, 1986, op. cit.

17. G.H. BousQuet précise à juste titre que les mots 'âda et 'urf ont le même sens, mais I'usage varie selon les régions. Et il est vrai que dans les pays du Maghreb le mot 'urf est d'un usage plus fréquent.

18. P. RICÆUR, Lecture (I) : autour du politique, Paris, Seuil, 1991, p. 180. 
M. Nachi

Justice et injustice du tanfîl : la part du légal et du conventionnel dans la construction sociale du sens ordinaire du juste

\section{Cette distinction}

fondamentale chez M. WALZER entre « égalité simple » et « égalité complexe » a fait l'objet d'une réflexion approfondie de la part des philosophes, sociologues et politistes dont les contributions ont été réunies dans un ouvrage collectif :

D. MILLER and M. WALZER (eds), Pluralism, Justice and Equality, Oxford, Oxford University Press, 1995 (cf. notamment D. MILLER, «Complex Equality », ibid., p. 197-225).

20. Ce que nous tentons d'analyser dans une autre étude. Cf. M. NACHI, « Le juste entre formalisme et substantialisme », Social Science Information/ Information sur les sciences sociales, 37 (4), 1998.

21. L. BOLTANSKI et L. THÉVENOT, De la justification : les économies de la grandeur, op. cit., p. 427 et passim.

22. Voir par exemple à ce sujet l'importante étude de V. LEE HAMILTON, « Social Consensus on Norms of Justice: Should the Punishment Fit the Crime? », American Journal of Sociology, 85 (5), 1980, p. 1117-1144. l'ont soutenu les théologiens, l'expiation de péchés légers, on peut dire que le tanfîl permet d'éviter ce qu'il pourrait y avoir d'injuste dans une distribution réglée par l'égalité arithmétique au profit d'une autre égalité qu'on pourrait qualifier, à la suite de M. Walzer, «d'égalité complexe». À la différence de l'égalité simple dont le principe est «à chacun la même chose», l'égalité complexe se révèle essentiellement réactive et corrective ${ }^{19}$. Le partage selon le procédé du tanfîl doit, pourrait-on dire, être inégalitaire pour être juste. II s'agit dans une certaine mesure d'une forme de justice «corrective» ou « rectificative » 20 , une justice qui permet de redresser et que l'on mobilise pour réaliser l'opération consistant à rechercher l'équité en ajoutant et en retranchant.

Dans le cas d'un partage foncier, ce procédé vise à introduire des paramètres propres à la nature du sol, à son emplacement, etc., en vue d'une répartition juste. II faut cependant souligner le caractère consensuel du tanfîl. En effet, on ne peut procéder au tanfîl que s'il y a approbation de toutes les parties concernées. Mais, en pratique, s'il n'y a pas de conflit ouvert, l'unanimité est souvent facile à trouver, dans la mesure où aucune partie n'a intérêt à refuser le tanfîl. Celui qui le refuse sera contraint à accepter la part la moins intéressante. C'est ce qui apparaît bien dans le récit, puisque les quatre frères ont très vite adopté le partage.

Nous revoilà ainsi au centre de la problématique: repérer, à travers les jugements sur la situation du tanfîl, là où il y a convergence et là où il y a divergence dans les jugements. Ceci afin de rattacher, dans la lignée de Boltanski et Thévenot, les différents types de jugement à des régimes pragmatiques de justification ou ordres de grandeur et de dégager le registre normatif invoqué pour chaque régime ${ }^{21}$. II s'ensuit que nous ne cherchons pas à trouver un consensus normatif autour de certains principes de justice, comme on le fait souvent dans les études de justice criminelle sur la détermination des sanctions pénales, où l'on demande aux sujets d'attribuer une peine juste en fonction de la gravité du délit commis et où I'on cherche celle qui fait le consentement de tous ${ }^{22}$. Au contraire, nous demandons aux enquêtés d'émettre des jugements sur le dénouement de l'affaire et de fournir des justifications à leurs jugements. Et ce sont les arguments qu'ils déploient à l'appui de ces jugements qui nous permettent de saisir en quoi ces jugements sont contrastés ou concordants.

Pour mener à bien notre enquête, nous avons élaboré un questionnaire destiné à être présenté aux gens pour recueillir leurs appréciations et jugements. En effet, à partir d'une affaire comme celle du tanfîl, nous leur demandons de procéder à la qualification du «dénouement de l'affaire » (juste, assez juste, assez injuste, injuste), de la nature du tort (grave, assez grave, pas grave du tout) et de l'action de chacun des protagonistes (très bien, assez bien, assez mal, très mal). Toutes ces qualifications ne sont pas sans 
renvoyer au sens moral, au sens de la justice de chacun et à la question cruciale de la coordination de l'action. Les qualifications du dénouement de l'affaire, de la nature du tort et de l'action doivent dès lors être créditées d'une dimension morale et pragmatique en ce sens qu'elles sont supposées donner une appréciation, une évaluation, de la «nature » bonne ou mauvaise de leurs raisons d'être. C'est, pour ainsi dire, leur teneur évaluative qui peut être révélatrice du sens du juste.

À travers le résultat statistique dont nous disposons, deux pôles de jugements se dessinent qui, sans être totalement opposés, se révèlent néanmoins sensiblement différents. L'agencement des arguments et justifications mis en œuvre par les personnes interrogées corrobore cette polarisation. Bien que les avis ne s'opposent pas terme à terme, des différences notables apparaissent et font surgir deux visions du juste. Mais, en définitive, ces deux visions trouvent leur ultime expression dans l'idée de compromis. Le premier pôle représente la position minoritaire qui défend une conception légale du juste et, dès lors, prône une primauté du formel sur le conventionnel. Le second pôle représente la position majoritaire qui défend une conception selon laquelle le conventionnel, en tant qu'il est communément partagé et socialement attesté, doit prendre le dessus. Nous allons, dans un premier temps, procéder à l'analyse des formes de justification de la première position (minoritaire) pour ensuite passer à celles de la seconde (majoritaire).

\section{L'adéquation du légal et du juste, ou le primat du formel sur le conventionnel}

\section{II.1. Le recours à la loi comme référentiel de justification au détriment du tanfîl}

Une minorité de personnes interrogées $(23,62 \%)$ considèrent le dénouement de cette affaire juste ou assez juste (cf. tableau $n^{\circ} 1$ ). Pour bien comprendre les significations que ces personnes donnent à l'expression «dénouement juste », il convient de mettre au jour les justifications et les arguments qui constituent le soubassement de leur jugement.

Pour la plupart de ces Tunisiens et Français, les affaires d'héritage en général et cette histoire en particulier sont toujours très compliquées ${ }^{23}$. II s'agit, dit-on d'une «affaire inextricable, [dans laquelle] tout le monde est perdant » (101) 24 . Comme le fait remarquer une enseignante, «les histoires d'héritage sont souvent sources de conflits 》 (117). Selon ces personnes, «seuls les tribunaux peuvent rétablir à peu près les droits de chacun » (95).

On note donc que ces personnes se placent sur un plan strictement légal où la loi positive, formelle, est la référence ultime.

23. Le caractère complexe, inextricable, de tout partage d'héritage a été souligné par presque la moitié des personnes interrogées. Certains parlent « d'imbroglio ».

24. Pour cette affaire, nous avons pu interroger 127 personnes : 49 Tunisiens et 78 Français. Pour faciliter la présentation des arguments des uns et des autres et éviter d'alourdir le texte, nous avons adopté le système de numérotation suivant : de 1 à 49 pour les Tunisiens et de 50 à 127 pour les Français. 
M. Nachi

Justice et injustice du tanfîl : la part du légal et du conventionnel dans la construction sociale du sens ordinaire du juste
Nous parlerons de référentiel légal pour marquer le caractère légaliste, formel et normatif de cette position. Mais il faut savoir dès maintenant que d'autres personnes, la majorité, préconisent autre chose que le droit positif pour asseoir leurs conceptions du juste, puisqu'elles font prévaloir le caractère conventionnel de l'idée de justice.

Pour les défenseurs de la position minoritaire, la décision de justice est en elle-même suffisante pour qualifier de juste le dénouement de cette affaire. Elle est juste parce qu'elle est conforme au droit. Car, affirme-t-on : «La loi a tranché selon la justice » (117), ou encore : «Le dénouement qui a été décidé [par le tribunal] est juste » (53). Un instituteur tunisien résume bien cette position : "La vérité c'est qu'il y a une "jalousie de succès" (hosd) entre ces frères. Tout d'abord, ils ont tous accepté le partage et après ils changent leurs idées pour partager cette terre à parts égales. C'est injuste. Mais la loi, c'est la loi. Comme il n'y a pas d'écrit entre eux, il faut dans toutes les circonstances accepter le nouveau partage bien qu'il soit injuste [...] $\gg(8)$.

Ce qui revient souvent chez les tenants de la position légaliste, c'est l'idée qu'un arrangement n'est pas envisageable, vu le caractère inextricable de ce genre d'affaires. Et ce sont cette inextricabilité et cette impossibilité d'aboutir à une solution négociée qui, selon ces personnes, rendent indispensable l'intervention de la loi. Ainsi, pour cet instituteur français, il faut « une entente entre tous les frères pour le règlement du litige sinon [il y a lieu d'appliquer le] droit en vigueur » (100). Pour cet agriculteur qui avait l'air de connaître «cette espèce d'engrenage », il faut « un dénouement où il n'y aurait aucune discussion, par respect de la mémoire du défunt ; or, poursuit-il, seul le droit peut faire taire tout le monde » (109). Il est donc clair que sans une décision judiciaire, formelle, il y aura toujours quelqu'un qui contestera et la querelle sera interminable.

II faut par ailleurs noter que ces personnes ne font jamais référence au tanfîl, sinon pour souligner ce qu'il représente de plus négatif, de plus caduc. Au plus est-il question d'un «système d'entente campagnard et désuet » (46) ou encore d'un «partage qui a été fait d'une façon un peu illégale et qui provoque un problème entre les frères puisque la part que Kamel a eu en plus est à l'origine du conflit » (24).

En revanche, ce qui est mis en avant et systématiquement invoqué par les tenants de la position légaliste, c'est l'écrit. Si la «force du droit » est considérée comme l'ultime recours pour arrêter les disputes, l'écrit est pour ces personnes le moyen nécessaire et incontournable pour les éviter. Avec un écrit, plus de contestation possible. Sans écrit, la porte reste ouverte aux disputes. L'écrit a, pour ainsi dire, une fonction préventive et sécurisante. Tirant les conclusions de cette «sombre affaire », une documentaliste dit : 
«II s'avère que l'écrit vous protège même de votre famille » (77).

De même, pour cette libraire, la nécessité d'avoir un écrit ne fait pas de doute et «même avec sa famille, il faut faire les affaires devant le notaire » (98) de telle sorte que chacun ait son contrat. Même avis chez ce fonctionnaire tunisien pour qui «tout partage doit être bien rédigé et signé par tous les intéressés afin que chacun sache ses droits et ses devoirs » (32).

\section{II.2. Une pluralité dans la qualification des torts}

Pour ceux qui accordent une place prépondérante à l'écrit, les quatre frères ont eu tort de ne pas procéder dès le début à l'enregistrement du partage. Ils doivent dès lors subir les conséquences de leur inconséquence. Pour ces personnes, il n'est pas important de voir qui est perdant et qui est gagnant, car il s'agit moins d'une affaire de personne que d'une question de principe. II se trouve qu'en l'occurrence, c'est Deli qui a le plus profité de ce dénouement et Kamel qui s'est mal tiré d'affaire, mais cela aurait pu être n'importe qui d'autre.

Ce type d'arguments converge avec l'idée, largement partagée par les défenseurs de la position minoritaire, selon laquelle le tort dans cette affaire n'est pas très grave.

Ainsi, parmi les 11 Français (sur 78) qui jugent juste (ou assez juste) le dénouement de cette affaire (cf. tableau $n^{\circ} 1$ ), aucun ne qualifie ce tort de «très grave»: 7 personnes le qualifient d'«assez grave » et les 4 autres de «pas grave du tout ». Mais, à la différence des Français, aucun Tunisien ne le qualifie de «pas grave du tout ».

Chez les Tunisiens, en effet, seuls quelques-uns (5/19) le qualifient de très grave; pour les autres (14/19), il est «assez grave ». Mais chez les uns comme chez les autres, le tort est moins considéré dans sa composante morale que dans sa dimension «civile», au sens juridique du terme. C'est en ce sens qu'il est souvent qualifié de «banal » ou de «courant», dans la mesure où il est très fréquent et en quelque sorte inévitable dès lors qu'on procède à un partage d'héritage et, a fortiori, si on le fait sans acte notarié.

On peut cependant repérer une contradiction, au moins apparente, entre la distribution des jugements chez les Tunisiens et chez les Français. En effet, tout laisserait à penser que, dans cette posture légaliste et formaliste, les derniers seraient plus portés que les premiers à se rallier ou se rabattre sur le droit, en raison de l'ancrage du système juridique dans leur société. Or, on constate que, sur l'ensemble des personnes qui défendent cette position, un tiers seulement est français.

Seuls les arguments des uns et des autres peuvent nous aider à expliquer ce contraste dans les jugements. On ne trouve toutefois ni dans les conversations, ni dans les résumés, des arguments qui 
M. Nachi

Justice et injustice du tanfîl : la part du légal et du conventionnel dans la construction sociale du sens ordinaire du juste se rapportent directement à ce contraste. Un détour par l'analyse des justifications qui fondent la détermination de la personne responsable (Q4) et de la victime (Q6) s'avère alors indispensable. À ce propos, force est de constater qu'une majorité de personnes interrogées, les deux nationalités confondues, considère que c'est Deli qui a le plus grand tort et voit en Kamel la victime (cf. tableaux $n^{\circ} 3$ et 4).

Cependant, seule une partie de la minorité qui nous intéresse ici (les 23,62 \%) peut se retrouver dans cette distribution. II s'agit des Français qui, malgré le fait qu'ils jugent le dénouement juste (ou assez juste), ne manquent pas de souligner que la responsabilité incombe à Deli et que c'est Kamel la victime.

Les Tunisiens qui ont émis le même jugement de justice (juste ou assez juste) ont une perception sensiblement différente quant à la qualification du responsable et de la victime. Pour la majorité d'entre eux (11/19), en effet, ce n'est pas Deli mais Kamel qui a le plus grand tort, même s'il est par ailleurs la victime. II faut cependant préciser qu'il s'agit de deux torts de nature différente: si le tort de Deli est d'avoir causé un dommage matériel à l'un de ses frères, celui de Kamel est (aux yeux des Tunisiens) d'avoir tenté de transgresser une «norme ancestrale ». On invoque ici deux référentiels normatifs différents: le droit d'une part, la coutume de l'autre. Son grand tort, nous dit-on, est d'avoir essayé d'enfreindre, de violer, une convention sociale qui stipule la norme suivante: « Ne jamais laisser un "étranger" (barânî) pénétrer la terre des ancêtres. » Comme l'affirme une jeune fonctionnaire, « vendre une terre provenant d'un héritage familial à un "étranger" à la famille est un outrage » (46).

Ce qui est reproché à Kamel, c'est d'avoir «juré de ne vendre sa part d'héritage à aucun de ses frères » (récit, A5). Même si ces derniers se trouvent à l'origine de l'injustice qu'il a subie (tîzlam), Kamel n'a pas à passer outre une convention qui préserve l'unité du patrimoine. Si Deli - et dans une certaine mesure Tarek - est jugé responsable de l'éclatement de l'unité de la famille, Kamel est jugé plus sévèrement parce qu'il a failli contribuer à l'éclatement de l'unité du patrimoine foncier, c'est-à-dire à sa dilapidation, ce qui est, aux yeux des Tunisiens, beaucoup plus grave et dans une certaine mesure impardonnable. Car la discorde au sein de la famille n'est jamais définitive, alors que la mise en cause de l'unité d'un patrimoine l'est toujours. Après une dispute, tôt ou tard, les frères trouveront la voie de la réconciliation : «Si ce n'est pas un jour de fête, ce sera un jour de deuil », dit un proverbe tunisien. Par contre, si l'unité du patrimoine est entamée et si un « étranger » est installé, la rupture est consommée et il n'y a plus rien à faire, plus de solution possible. Pour vendre une part d'héritage, explique cette même fonctionnaire déjà citée, «il faut voir le prix que propose [un acheteur] "étranger" et vendre [à ce 
prix] à un membre de la famille » (46), ceci afin que le prix soit Droit et Société 39-1998 juste et que le vendeur ne soit pas lésé. Pour un autre fonctionnaire, même si les relations entre les quatre frères «ne sont pas bien fondées » parce qu'il y a eu recours devant la justice, il n'en reste pas moins que «le côté positif est représenté dans cette histoire par Rachid qui n'a pas accepté de laisser entrer un "étranger" sur leurs terres $\gg(2)$.

Rachid est dès lors présenté comme le «sauveur » de la situation, comme celui qui a osé intervenir «judicieusement » pour préserver l'unité du patrimoine.

Évidemment les Français ont un autre jugement sur l'attitude de Rachid. Pour eux, il incarne la personne la plus riche et la plus forte, celle qui a pu infléchir la volonté de son frère pour consolider ses propres intérêts. Rachid, dit une étudiante, «est le plus riche des quatre frères [qui] intente une action en justice et oblige Kamel à lui vendre ce terrain » (76). Or, comme le fait remarquer un gérant d'entreprise, «chacun fait ce qu'il veut de son bien, quelle que soit la provenance » (112), et on n'a pas à lui imposer un acheteur, même s'il s'agit de l'un de ses frères.

Au total, il faut bien reconnaître qu'aussi bien les Tunisiens que les Français invoquent des normes, des valeurs et des catégories juridiques pour montrer le bien-fondé de ce qu'ils considèrent comme juste dans cette affaire. Nous venons de voir que dans cette posture minoritaire, les Tunisiens sont majoritaires. Puisqu'il en est ainsi, on serait tenté de penser qu'ils sont plus légalistes ou formalistes que les Français dans leur perception du sens de la justice. Les choses sont cependant plus subtiles. Pour s'en rendre compte, il convient de définir la nature du référent normatif auquel les uns et les autres recourent pour justifier leurs points de vue.

\section{II.3. Deux ordres de grandeur au fondement des référentiels normatifs : grandeur domestique et grandeur marchande}

Les développements précédents font ressortir deux références fondamentales à deux ordres de grandeur, au sens que donnent Boltanski et Thévenot à cette notion ${ }^{25}$. Alors que les justifications des Français s'organisent exclusivement autour d'une forme de généralité marchande, celles des Tunisiens s'orientent vers une forme de général ité domestique.

Bien qu'ils défendent une position légaliste semblable à celle des Français, les Tunisiens font appel à des formes de justification d'une nature différente. S'ils ne manquent pas d'utiliser des arguments de type légal et de recourir à des principes de nature marchande pour nourrir leurs justifications, en y regardant de plus près, on constate que ces justifications ainsi que les principes qui les fondent ne sont que partiellement de nature marchande.

25. L. BoltANSKI et L. THÉVENOT, De la justification : les économies de la grandeur, op. cit. p. 26. 
M. Nachi

Justice et injustice du tanfîl : la part du légal et du conventionnel dans la construction sociale du sens ordinaire du juste

26. II se trouve que, dans ce cas précis, il n'y a pas inadéquation entre ce qu'affirme la coutume et ce que stipule la loi écrite et ceci pour la simple raison que celle-ci n'est en définitive que la codification de celle-là. Pour un aperçu sur l'avènement du travail de codification dans le domaine des droits civils en Tunisie et sur son approche pragmatique, voir M. ZINE, «Centenaire de la codification en Tunisie : le Code des Obligations et des Contrats », dans B. BEIGNIER (dir.), La codification, Paris, Dalloz, 1996, p. 187-195. S'agissant de la codification en général, la réflexion de $P$. BOURDIEU demeure essentielle. Voir entre autres, P. BOURDIEU, « Habitus, code et codification », Actes de la recherche en sciences sociales, 64, 1986, p. 40-44.

27. Il convient de rappeler à cet égard que les juristes

définissent souvent la coutume, entre autres, par son aspect

«matériel ».
L'inventaire des ordres de justification fait apparaître chez les Tunisiens la prépondérance du dispositif domestique au détriment de l'espace référentiel marchand, ce qui s'explique par le fait que les Tunisiens font prévaloir les pratiques coutumières, les valeurs conventionnelles sur les textes juridiques formels ou les intérêts individuels. À la différence des Français pour qui c'est la loi, la légalité et la liberté de disposer de ses biens qui doivent prendre le dessus, pour les Tunisiens, au contraire, c'est la coutume, comme potentiel normatif communément partagé, qui doit prendre le dessus.

Rappelons que Rachid a intenté une action en justice contre Kamel en alléguant son droit de préemption et qu'il a eu gain de cause en vertu d'une loi en vigueur. Et ce qu'on reproche à Kamel, c'est d'avoir cherché un acheteur «étranger » pour lui vendre sa part d'héritage, alors qu'il savait pertinemment que cela est contraire aux us et coutumes de sa communauté. En fin de compte, le grief qu'on lui fait, c'est moins le manque de respect pour la loi que sa maladresse à tenter de transgresser une norme sociale ancestrale, une coutume, dont il n'ignore aucunement l'importance ${ }^{26}$.

Et ce sont ces références simultanées à la coutume, au détriment de la loi, aux proches (frères), au détriment de lointains (étranger), à l'unité de la famille et du patrimoine, au détriment de leur atomisation, qui nous autorisent à ramener le répertoire de justification des Tunisiens à la nature domestique plutôt qu'à la nature marchande. Ces ressources domestiques de justification, que les Tunisiens mettent en œuvre pour trouver un accord sur la nature du juste dans cette situation, les amènent à placer la forme de généralité domestique au dessus de la forme de généralité marchande bien prépondérante dans les justifications des Français.

\section{L'inadéquation du légal et du juste, ou le primat du conventionnel sur le formel}

\section{III.1. Le recours au tanfîl comme référentiel de justification au détriment de la loi}

À la différence de la minorité dont on vient de parler, la majorité des personnes interrogées $(76,37 \%)$ jugent le dénouement de cette affaire assez injuste (25,98\%) ou injuste (50,39\%) (cf. tableau $\mathrm{n}^{\circ} 1$ ). Il faut d'emblée préciser que les implications juridiques et morales des formes de justification qui sous-tendent l'accord sont ici différentes de celles exposées plus haut. À la différence de la position légaliste, où l'accent est mis sur le caractère égalitaire et formel de la règle de droit (Français) ou sur la prééminence d'une norme coutumière (Tunisiens), on fait valoir ici le caractère conventionnel des principes de partage et, en quelque sorte, le contenu de la norme, son caractère «matériel » pourrait-on dire 27 . 
En effet, pour toutes les personnes qui nous concernent ici, il ne fait pas de doute que le premier partage est juste ${ }^{28}$. À la différence de la minorité, qui ne fait aucunement mention du tanfîl, ici tout le monde en parle avec, même, un petit air nostalgique. Aussi bien les Tunisiens que les Français ne manquent pas de rappeler que c'est un partage conventionnellement consenti, fait «selon une méthode traditionnelle», selon «la coutume», selon «la tradition », ou tout simplement que c'est un «partage à l'ancienne mode », pour dire qu'il fait intervenir des considérations inhérentes au bien à partager, c'est-à-dire des «critères objectifs » (64), et que, en tant que tel, il est juste. Voici comment une enseignante tunisienne résume ces différents aspects : «II s'agit d'un problème de partage d'héritage juste selon les anciennes traditions et les valeurs qui ont régné autrefois et qui semble de nos jours injuste selon les normes du tribunal. C'est pourquoi il y a eu un repartage qui est en faveur de Deli mais qui est une catastrophe pour le pauvre Kamel qui était victime de l'égoïsme et du « désir de possession » [raghba] de Deli » (18). Une enseignante française dit à sa façon, avec un peu plus de distance peut-être, ce qu'elle pense du tanfîl : «C'est une méthode apparemment assez juste qui prend en compte de nombreux paramètres mais qui a pour inconvénient de se pratiquer couramment sans acte d'enregistrement » (107). Les deux personnes mettent l'accent sur le caractère «juste» ou «assez juste » de la méthode initialement utilisée pour partager I'héritage, même si l'enseignante française ne manque pas de relever l'inconvénient d'un partage conventionnel qui n'est pas consigné par écrit. Il faut souligner à ce propos que, si on parle de l'écrit, c'est pour lui attribuer la fonction conservatoire d'une coutume jugée juste, en ce sens qu'elle permet l'utilisation de «critères objectifs » lors d'un partage. Nous verrons plus loin comment il est fait ici recours à l'écrit, non pas tant pour qu'il prenne le dessus sur la coutume, comme c'est le cas chez les défenseurs de la position légaliste, mais au contraire pour la sauvegarder.

L'énoncé «ce qui est légal peut être injuste » apparaît comme le leitmotiv de la plupart des personnes défendant le point de vue ici développé. Cet énoncé s'applique parfaitement au tanfîl, en ce sens qu'on a là une pratique de partage jugée juste mais qui se trouve ignorée et désavouée par le droit. La norme juridique s'est révélée injuste dans son refus d'intégrer des critères conventionnels d'attribution, socialement partagés, qui ont pour particularité de permettre la réalisation d'un partage juste, c'est-à-dire un partage permettant d'attribuer à chacun son dû en tenant compte de la nature et des particularités du bien à distribuer. Nous pouvons à cet égard parler, à la suite de J.-Ch. Maymat, de «l'effet injuste d'un jugement juste 29 ».
Droit et Société 39-1998

28. Il faut toutefois mentionner l'exception d'une personne, singulièrement pessimiste, qui ne voit à cette affaire aucune issue possible. Elle dit : «II ne peut y avoir de dénouement juste dans cette histoire qui est un conflit de cultures : les deux cultures coutumière et moderne ont leur propre justice et les deux sont incompatibles. C'est une histoire de fous ! insoluble, d'autant que certains ne me paraissent pas de bonne foi » (120).

29. J.-Ch. MAYMAT, « L'effet injuste d'un jugement juste », dans M-A. FRISON-ROCHE et W. BARANĖS (dir.), De l'injuste au juste, Paris, Dalloz, 1997, p. 90. 
M. Nachi

Justice et injustice du tanfîl : la part du légal et du conventionnel dans la construction sociale du sens ordinaire du juste
30. Précisons toutefois que, dans la question $n^{\circ} 4$ qui permet aux enquêtés de dire quel est le personnage du récit qui «a le plus grand tort », nous avons omis de proposer le nom du narrateur. Ils avaient donc le choix entre trois frères seulement et pourtant certains n'ont pas hésité à rajouter le nom de Tarek ou à nous le signaler. Il ressort également des entretiens - et même du récit - que Tarek doit être considéré, avec Deli, comme étant à l'origine du litige.
Comme le dit une jeune femme, «l'évolution des critères de transmission du foncier d'utilitaires à arithmétiques » (66) a eu pour conséquence une décision judiciaire totalement injuste. Ou encore : «C'est une histoire où la légalité avalise une injustice » (57). La disqualification de la loi, son désaveu, est ici synonyme de la prééminence de la composante conventionnelle des principes de justice.

\section{III.2. La qualification du fautif et de la victime}

Bien que l'attitude du juge ne soit pas louée, ni sa grandeur célébrée, il n'en reste pas moins que les enquêtés ne lui imputent aucune responsabilité, même s'ils considèrent par ailleurs qu'il a «mal jugé ». Pour eux, il faut remonter à l'origine du conflit pour pouvoir déterminer celui qui a tort et celui qui a raison.

À l'évidence, la responsabilité est à chercher du côté de celui qui a déclenché le conflit. Du côté, nous dit-on, « de celui qui est à l'origine du mal ». Et là, le résultat statistique est sans ambiguïté, dans la mesure où $76,38 \%$ des personnes interrogées considèrent que c'est Deli et dans une moindre mesure Tarek qui ont le plus grand tort dans cette affaire (cf. tableau $\left.n^{\circ} 3\right)^{30}$. C'est parce qu'ils étaient à l'origine du déclenchement de ce «malheureux » litige qu'il faut leur imputer une part non négligeable de responsabilité.

Ainsi, pour certains Tunisiens, Tarek aurait dû accorder un droit de passage à son frère Deli, moyennant une compensation matérielle par exemple, pour éviter les hostilités (45). Pour certains Français, «Deli et Tarek auraient pu s'entendre pour trouver une solution et éviter tous les tracas!» (58). Et on ajoute: «S'ils s'étaient arrangés soit pécuniairement [soit] en échangeant des terres, la famille serait toujours unie » $(74,75)$. Pour « une chose insignifiante », le désaccord entre eux, souligne-t-on, « va engendrer de multiples déboires qui mettent en cause le partage initial » (73). Tout cela est d'autant plus aberrant, ajoute une étudiante, «que finalement le problème entre ces deux frères (passage de la charrette) n'est pas réglé » (99).

Mais si les deux frères sont considérés comme ayant une responsabilité commune parce qu'ils étaient à l'origine du différend (la mise en cause du premier partage), c'est finalement Deli qui est le plus sévèrement jugé. Deli cumule, si l'on peut dire, une autre responsabilité : son «égoïsme » et son «manque d'honnêteté intellectuelle» (57) lors du second partage. Pour beaucoup de Tunisiens, «Deli n'aurait dû jamais accepter la somme d'argent » (30) ou, ce qui revient au même, «il aurait dû acquitter son frère Kamel du remboursement» (11). Le même point de vue est défendu par des Français, pour qui «Deli doit refuser l'argent de Kamel, puisqu'il était au départ d'accord sur le partage initial » 
(49). C'est pourquoi, ajoute-t-on, «il doit renoncer à la somme qui lui a été attribuée par le tribunal $\gg(70)$.

De telles affirmations laissent penser qu'à partir du moment où l'on considère qu'une décision judiciaire est injuste et qu'on a la possibilité de la contourner, il ne faut surtout pas hésiter à le faire. Car, finalement, ceci permet de rétablir la justice et de «corriger » le droit. En l'occurrence la puissance publique ne peut pas contraindre Deli à accepter l'argent. Et sachant que son frère va lui verser injustement cet argent, Deli doit, par conséquent, refuser de l'encaisser. Et même si on l'y oblige, ajoute une retraitée, il peut prendre l'argent et ensuite, plus tard, le rendre à son frère. Car, la « conscience morale » (damîr) ne nous permet pas d'accepter cette injustice.

\section{III.3. Justesse de l'écrit, justice du compromis}

On aura compris l'importance qu'accordent les enquêtés à un système normatif conventionnellement établi dont le souci majeur est de prendre en compte différents paramètres afin d'aboutir à une décision qui ne serait pas la simple application de la règle de droit, mais la résultante d'une pluralité de facteurs normatifs. Comme le soutient une enseignante, «la solution peut être "horsla-loi" [et] ménager les liens fraternels, dans un système d'échanges consenti » (54).

Et, à l'évidence, une écrasante majorité de nos enquêtés insiste sur la nécessité d'un compromis. Si les quatre frères en sont arrivés là, c'est, affirme-t-on, parce qu'il y a eu malheureusement absence de compromis et parce que «suite à tout héritage, un égoïsme humain s'installe dans toute la famille et chacun veut tirer la corde de son côté » (1). Et on ajoute : «L'argent et les biens ont toujours été à l'origine de conflits entre les parents. Lorsqu'il s'agit de ses propres intérêts, le frère devient le pire ennemi du sien » (44).

Or, dans ce genre de situation, il faut que chacun mette du sien et dépasse ses propres intérêts immédiats pour «regarder audelà » et faire passer l'intérêt de la famille, son unité, avant ses propres «calculs égoïstes». Dès lors, affirme une étudiante, «il faut que les frères oublient leurs intérêts pécuniaires et respectent le pacte passé avec le père, juste, et s'entendent en discutant » (119). C'est pourquoi les frères n'auraient jamais dû aller devant les tribunaux (51). Pour éviter cette «brouille familiale » (56), cette «fraternelle trahison » (83), les frères auraient dû tenir leur parole et rester fidèles au partage d'origine. C'est parce qu'ils ont manqué à leur parole que les frères ont fait subir à l'un des leurs une « fâcheuse » injustice et que l'unité de la famille a volé en éclats.

C'est là qu'apparaît pour certains la nécessité d'avoir un texte écrit et non une «parole légère ». L'écrit aurait pu servir à rendre 
M. Nachi

Justice et injustice du tanfîl : la part du légal et du conventionnel dans la construction sociale du sens ordinaire du juste

31. D. HowES, «De l'oralité et de la lettre de la loi », Droit et Société, 32, 1996, p. 27-49.

32. M. Detienne, « L'espace de la publicité : ses opérateurs intellectuels dans la cité $»$, dans M. Detienne (dir.), Les savoirs de l'écriture en Grèce ancienne, Lille, PUL, 1992, p. 29-81.

33. G. LENCLUD, «L'opposition coutume-droit dans les systèmes de reproduction », art. cité,

p. 17.

34. II faut noter que, depuis les Grecs, I'opposition entre l'oral et l'écrit ne tient pas et le sens de nomos, dont on a parlé plus haut, est propre à la cité. Comme l'écrit J .-L. AMSELLE : « La catégorie de nomos, en effet, n'apparaît pas liée de façon absolue à un mode d'enregistrement écrit mais plutôt à l'existence d'une isonomie et d'une justice distributive. », cf. J.-L. AMSELLE, «Le droit contextualisé », Cahiers d'études africaines, 124, XXXI (4), 1991, p. 553. Voir, sur ces différents aspects de I'écriture en Grèce ancienne, M. DETIENNE (dir.), 1992, op. cit. 35. M. NACHI, 1996, op. cit. irrévocable un partage initial juste et à veiller au respect de la parole donnée. Le contraste entre l'écrit (enregistrement) et l'oral (parole donnée) s'estompe ici au profit d'une complicité nécessaire qui rend caduque toute opposition rigide entre droit et coutume ${ }^{31}$. Montrant comment l'écrit répond aux exigences d'un « espace de la publicité », selon l'expression de M. Detienne ${ }^{32}$, et comment la parole aussi bien que l'écrit sont soumis aux exigences de codification, G. Lenclud ne manque pas de souligner que «le langage écrit est presque toujours considéré dans ses rapports avec la parole, jamais pour lui-même. Qu'on lui assigne, quasi immanquablement, une position seconde par rapport à la parole : la raison d'être de l'écriture serait de la représenter, de la conserver 33 ». Entre l'oral et l'écrit, il y a donc bien davantage continuité que rupture. Les mettre en opposition refléterait une vision simplificatrice, positiviste, sans véritable fondement ${ }^{34}$. Ces affirmations coïncident bien avec le point de vue de nos enquêtés, avec le sens commun qui considère que seul l'écrit aurait pu empêcher la dégradation de cette situation.

On fait par conséquent prévaloir l'écrit pour préserver ce partage et non pour le mettre en cause, comme l'a fait la décision du tribunal. L'écrit est ainsi appelé au secours d'une coutume, le tanfîl, pour faire respecter sa justesse. L'écrit s'avère alors important pour éviter les disputes, car «lorsqu'il y a partage des biens, il y en a toujours un qui fait des histoires » (125). Comme le dit ce maître d'application tunisien : «On ne doit pas avoir une part d'héritage sans l'enregistrer car il n'y a plus de parole, même entre les frères et sœurs, parce qu'en notre temps chacun se débrouille pour gagner sa vie »; et il conclut par ce proverbe : «Si le frère était utile [compréhensif] pour son frère, personne ne pleurerait la mort de son père » [loukân al-khou yenfa'a khûh, mâ yebkî had 'ala bûh] (6). Et ce libraire français d'ajouter : « Même en famille, ne jamais faire confiance à la parole. Seuls les écrits restent et sont incontestables. Dans toutes les familles du monde, on trouve ce genre de problèmes, des plus pauvres aux plus riches » (97).

Ainsi, ces différentes personnes regrettent que les frères n'aient aucun moyen pour faire respecter le premier partage. L'écrit aurait pu être le moyen idéal et incontestable. Comme le dit cette secrétaire : «Les paroles s'envolent, les écrits restent. Si tout avait été fait dans les règles, il n'y aurait pas eu de problèmes dans la manière [dont] a été partagée la terre » (111).

\section{Conclusion}

Au terme de cette incursion dans les arguments des uns et des autres, on ne peut s'empêcher d'être frappé par la corrélation très étroite entre, d'une part, la thèse que nous avons défendue d'un point de vue strictement théorique dans une étude antérieure ${ }^{35}$ et, 
d'autre part, les résultats empiriques dont nous venons d'analyser en profondeur la substance. À l'instar de cette position visant à dépasser la fausse alternative entre une conception procédurale de la justice, défendue entre autres par Rawls et Habermas, et une conception substantielle, préconisée par les auteurs dits communautariens, ces résultats nous montrent qu'il n'y a pas, d'un côté, une position légaliste, formaliste et normativiste qui serait l'apanage des Français et, de l'autre, une conception conventionnelle qui serait réservée aux seuls Tunisiens, mais qu'il y a, entre les deux, sinon interpénétrations, du moins rapprochements. II y a même chez certaines personnes, en arrière-plan des deux positions, l'idée d'un dépassement de ces deux approches au profit d'une solution prenant ses fondements dans l'une comme dans l'autre, sans exclusive. La construction d'un compromis serait à leurs yeux le moyen par excellence de parvenir à cette fin.

Nous avons à cet égard tenté de montrer comment, à travers la mobilisation des divers registres de justification, aussi bien les Tunisiens que les Français oscillent entre les deux positions, opèrent entre elles un va-et-vient constant, même si à la fin il y a chez les uns comme chez les autres tantôt prééminence de l'une, tantôt prévalence de l'autre. Entre les deux se distribue une multitude d'attitudes et de jugements traduisant la pluralité du sens de la justice. II faut dès lors relever le lien évident et, à certains égards, l'interférence entre les conceptions légaliste et conventionnelle. Cela nous conduit à suggérer que le juste se situerait entre les deux.

En poursuivant l'analyse des arguments et justifications propres à chacune des deux positions, nous avons en effet pu voir comment les personnes s'efforcent de se frayer des chemins aux confins de ces deux ordres de grandeur. L'argumentation déployée lors des opérations de jugement nous renvoie tour à tour à la posture légaliste et aux principes conventionnels, se propage d'un pôle à l'autre, pour finalement nous inviter à focaliser notre attention sur la nécessité de construire, dans de pareilles situations conflictuelles, des compromis. Ni le recours à la simple légalité formelle ni la référence à la seule dimension conventionnelle de la règle de justice ne suffisent à rendre compte de la configuration complexe de la justice ${ }^{36}$.
Droit et Société 39-1998

36. Je tiens à remercier particulièrement Abderrazak Moulay Rchid, doyen de la Faculté de droit de Rabat, et Alain Roussillon, directeur de I'Institut de recherches sur le Maghreb contemporain (IRMC). $\mathrm{J}$ 'exprime également toute ma gratitude à André Robinet pour sa lecture critique et sa perspicacité. 
M. Nachi

Justice et injustice du tanfîl : la part du légal et du conventionnel dans la construction sociale du sens ordinaire du juste
Tableau $n^{\circ} 1$ J ugements sur la manière dont cette affaire s'est dénouée

\begin{tabular}{|l|c|c|c|c|c|}
\hline Nationa./ Q2 & juste & assez juste & assez injuste & injuste & Total \\
\hline Tunisiens & 13 & 6 & 7 & 23 & 49 \\
\hline Français & 2 & 9 & 26 & 41 & 78 \\
\hline Total col. & 15 & 15 & 33 & 64 & 127 \\
\hline Total $\%$ & $11,81 \%$ & $11,81 \%$ & $25,98 \%$ & $50,39 \%$ & $100 \%$ \\
\hline
\end{tabular}

Tableau $\mathrm{n}^{\circ} 2$

Qualification de la gravité du tort par des Tunisiens et des Français

\begin{tabular}{|l|c|c|c|c|}
\hline Nationa./ Q5 & très grave & assez grave & pas grave & Total \\
\hline Tunisiens & 22 & 25 & 2 & 49 \\
\hline Français & 24 & 48 & 6 & 78 \\
\hline Total col. & 46 & 73 & 8 & 127 \\
\hline Total $\%$ & $36,22 \%$ & $57,48 \%$ & $6,30 \%$ & $100 \%$ \\
\hline
\end{tabular}

Tableau $\mathrm{n}^{\circ} 3$

La personne qui a le plus grand tort dans cette affaire

\begin{tabular}{|l|c|c|c|c|}
\hline Nationa./ Q4 & Kamel & Deli & Rachid & Total \\
\hline Tunisiens & 11 & 35 & 3 & 49 \\
\hline Français & 4 & 62 & 12 & 78 \\
\hline Total col. & 15 & 97 & 15 & 127 \\
\hline Total $\%$ & $11,81 \%$ & $76,38 \%$ & $11,81 \%$ & $100 \%$ \\
\hline
\end{tabular}

Tableau $n^{\circ} 4$

La victime dans cette affaire

\begin{tabular}{|l|c|c|c|c|c|}
\hline Nationa./ Q6 & Kamel & Deli & Rachid & $\begin{array}{l}\text { Kamel }+ \\
\text { Deli }\end{array}$ & Total \\
\hline Tunisiens & 39 & 2 & 6 & 2 & 49 \\
\hline Français & 73 & 4 & 1 & 0 & 78 \\
\hline Total col. & 112 & 6 & 7 & 2 & 127 \\
\hline Total \% & $88,19 \%$ & $4,72 \%$ & $5,51 \%$ & $1,57 \%$ & $100 \%$ \\
\hline
\end{tabular}

